\title{
Effect of Metformin on Mitophagy in Newly Diagnosed patients with T2DM: A Randomized, Placebo-Controlled Study
}

\section{Shipra Bhansali ${ }^{1}$, Anil Bhansali ${ }^{2}$, Veena Dhawan ${ }^{1^{*}}$}

1 Department of Experimental Medicine and Biotechnology, ${ }^{2}$ Department of Endocrinology, Postgraduate Institute of Medical Education and Research (PGIMER), Chandigarh, INDIA

\section{INTRODUCTION}

Besides insulin deficiency and insulin resistance, patients with T2DM exhibit abnormalities in cell reparative process like mitophagy (selective autophagy of mitochondria). Metformin is widely prescribed as a first-line drug in the management of T2DM. It is a potent insulin sensitizer and exerts its pleiotropic effects via AMPK, however, it's effect on mitophagy remains unexplored.

\section{AIM \& OBJECTIVES}

The present study aims to elucidate the effect of metformin on mitophagy in newly diagnosed patients with T2DM (NDT2DM). Following parameters were assessed at baseline and 3 months post follow-up

- Mitochondrial stress indices including mitochondrial ROS (mtROS) content and mitochondrial membrane potential (MMP)

Translational expression of mitophagy-related markers (PINK1, PARKIN, MFN2, NIX, LC3-II and LAMP-2)

Quantification of damaged mitochondria in the study subjects by transmission electron microscopy (TEM)

\section{METHODS}

- Forty five drug-naïve NDT2DM patients with a duration of diabetes $<6$ months and $\mathrm{HbA}_{1 \mathrm{c}}$ levels $7-8 \%(53-63.9 \mathrm{mmol} / \mathrm{mol})$ were randomized into 3 groups ( $\mathrm{n}=15$ each):

$>$ Group-I received metformin at a dose of $2 \mathrm{~g}$ /day in divided doses

$>$ Group-II received voglibose at a dose of $0.3 \mathrm{mg}$ thrice/day

$>$ Group-III received placebo at a dose of $2 \mathrm{~g} /$ day

$>$ All study subjects were advised to follow lifestyle modifications to achieve a target $\mathrm{HbA}_{1 \mathrm{C}}$ level $\leq 6.5 \%(47.5 \mathrm{mmol} / \mathrm{mol})$

Voglibose was used as an active comparator to eliminate the effect of glucotoxicity on mitophagy, with respect to metformin

Anthropometry, parameters of glucose-insulin homeostasis were assessed at baseline and after 12 weeks of treatment

- Mitochondrial oxidative stress indices, translational expression studies of mitophagy-related genes (PINK1, PARKIN, MFN2, NIX, LC3-II, and $L A M P-2)$ and TEM studies were performed in peripheral blood mononuclear cells (PBMCs)

\section{RESULTS}

\begin{tabular}{|c|c|c|c|c|}
\hline \multicolumn{5}{|c|}{ Baseline characteristics of NDT2DM Subjects } \\
\hline Variables & $\begin{array}{c}\text { Metformin } \\
(\mathrm{n}=15)\end{array}$ & $\begin{array}{c}\text { Voglibose } \\
(\mathrm{n}=15)\end{array}$ & $\begin{array}{c}\text { Placebo } \\
(\mathrm{n}=15)\end{array}$ & P value \\
\hline Age (years) & $47.8 \pm 7$ & $45.5 \pm 8.7$ & $44.1 \pm 8.4$ & 0.345 \\
\hline Gender (M:F) & $7: 8$ & $6: 9$ & $8: 7$ & - \\
\hline Ht. (cms) & $155.0 \pm 9.5$ & $157.8 \pm 9.2$ & $156.2 \pm 8.1$ & 0.708 \\
\hline Wt. (Kgs) & $70.1 \pm 11.1$ & $74.4 \pm 9.3$ & $72.9 \pm 8.3$ & 0.468 \\
\hline BMI $\left(\mathrm{kg} / \mathrm{m}^{2}\right)$ & $29.2 \pm 3.3$ & $29.9 \pm 3.4$ & $29.9 \pm 3.5$ & 0.824 \\
\hline WC (cms) & $95.7 \pm 5.3$ & $99.3 \pm 6.5$ & $97 \pm 8.6$ & 0.361 \\
\hline Body Fat (\%) & $35.6 \pm 10.6$ & $37.5 \pm 9.3$ & $39.2 \pm 8.9$ & 0.596 \\
\hline Systolic BP & $126.0 \pm 12.9$ & $127.3 \pm 10.9$ & $122.0 \pm 10.8$ & 0.434 \\
\hline Diastolic BP & $82.0 \pm 8.6$ & $88.0 \pm 7.4$ & $80.4 \pm 7.1$ & $0.028^{*}$ \\
\hline FPG (mg/dl) & $138.4 \pm 17.2$ & $140.9 \pm 21.4$ & $160.2 \pm 22.1$ & $0.010^{* * *}$ \\
\hline $2 \mathrm{hPG}(\mathrm{mg} / \mathrm{dl})$ & $255.4 \pm 57.5$ & $241.4 \pm 58.9$ & $299.5 \pm 58.9$ & $0.025^{*}$ \\
\hline $\mathrm{HbA}_{1 \mathrm{c}}(\%)$ & $7.7 \pm 0.4$ & $7.6 \pm 0.4$ & $7.72 \pm 0.4$ & 0.664 \\
\hline FPI $(\mu \mathrm{IU} / \mathrm{ml})$ & $13.7 \pm 5.3$ & $15.9 \pm 6.0$ & $13.7 \pm 5.1$ & 0.438 \\
\hline HOMA-IR & $4.8 \pm 2.2$ & $5.5 \pm 2.1$ & $5.6 \pm 2.8$ & 0.588 \\
\hline HOMA- $\beta \%$ & $67.2 \pm 27.7$ & $75.3 \pm 35.9$ & $50.3 \pm 14.0$ & 0.084 \\
\hline CHOL $(\mathrm{mg} / \mathrm{dl})$ & $188.5 \pm 35.6$ & $205.0 \pm 40.7$ & $204.0 \pm 25.5$ & 0.355 \\
\hline LDL-C (mg/dl) & $125.1 \pm 33.8$ & $137.7 \pm 31.0$ & $133.7 \pm 27.5$ & 0.549 \\
\hline TG (mg/dl) & $141.0 \pm 41.9$ & $159.0 \pm 49.0$ & $160.0 \pm 66.6$ & 0.559 \\
\hline HDL-C (mg/dl) & $41.9 \pm 6.4$ & $41.2 \pm 6.6$ & $46.6 \pm 13.5$ & 0.277 \\
\hline
\end{tabular}

\section{$\Delta$ change in biochemical characteristics following treatment}

\begin{tabular}{|c|c|c|c|c|c|c|}
\hline Variables & $\begin{array}{l}\text { Metformin } \\
\text { group }\end{array}$ & $\begin{array}{l}\text { Voglibose } \\
\text { group }\end{array}$ & $\begin{array}{l}\text { Placebo } \\
\text { group }\end{array}$ & $\begin{array}{l}\text { Met vs. } \\
\text { Vogli } \\
\text { P value }\end{array}$ & $\begin{array}{l}\text { Met vs. } \\
\text { Plac } \\
\text { P value }\end{array}$ & $\begin{array}{c}\text { Vogli vs. } \\
\text { Plac } \\
\text { P value }\end{array}$ \\
\hline$\Delta \mathrm{Wt}$. (Kgs) & $-4.6 \pm 3.4$ & $-3.9 \pm 2.7$ & $-2.6 \pm 2.9$ & 1.000 & 0.277 & 0.863 \\
\hline$\Delta \mathrm{HbA}_{1 \mathrm{c}}(\%)$ & $-1.3 \pm 0.5$ & $-1.2 \pm 0.5$ & $+0.2 \pm 0.9$ & 1.000 & $0.0001 * * *$ & $0.0001^{* * * *}$ \\
\hline$\triangle$ HOMA-IR & & & & 1.000 & 1.000 & 1.000 \\
\hline$\triangle$ HOMA- $\beta \%$ & $+7.5 \pm 32.9$ & $+4.3 \pm 28.4$ & $+1.8 \pm 24.9$ & 1.000 & 1.000 & 1.000 \\
\hline
\end{tabular}
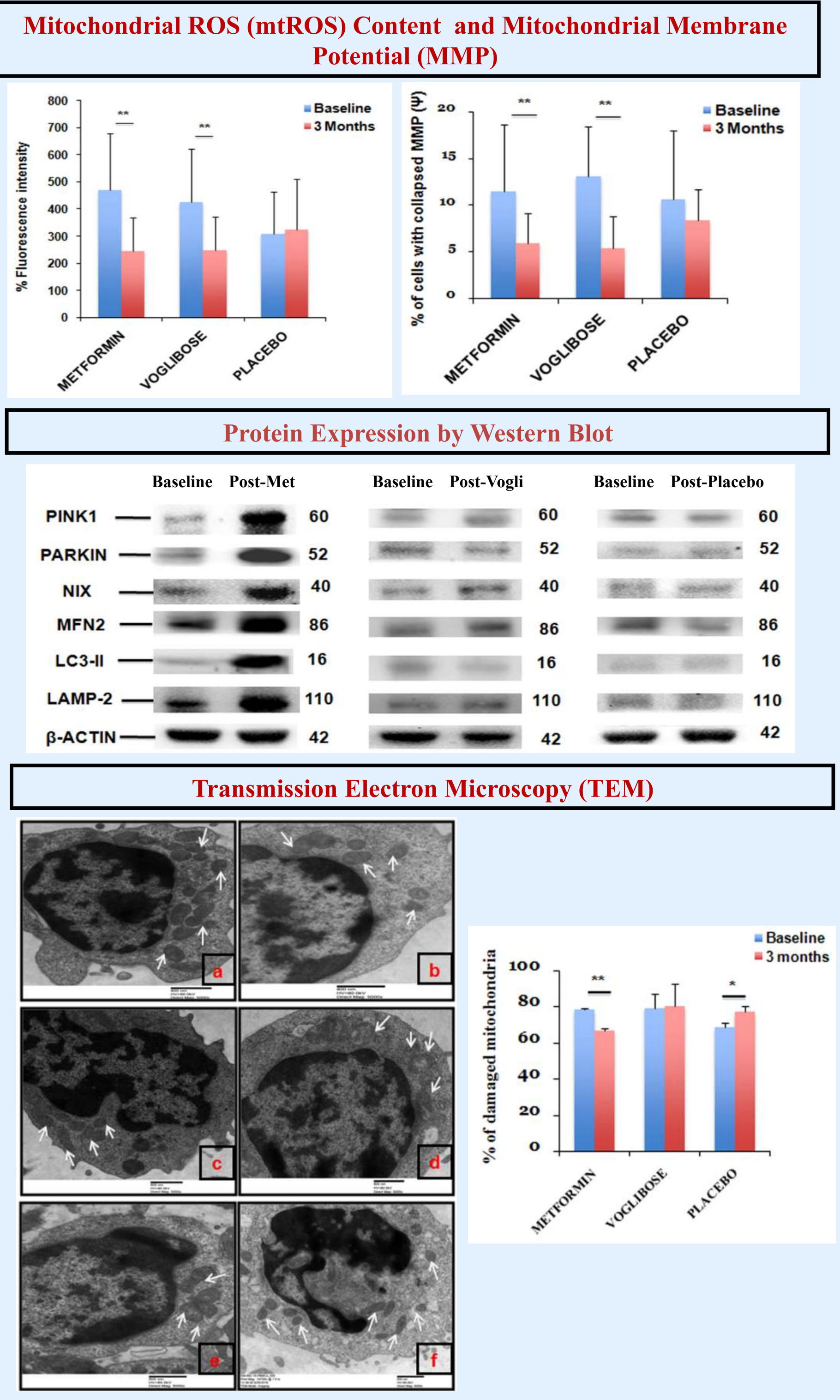

\section{DISCUSSION AND CONCLUSION}

- Both metformin and voglibose treatment showed a similar efficacy towards the reduction in plasma glucose and $\mathrm{HbA}_{1 \mathrm{c}}$ levels.

- Weight loss was comparable among all the study subjects at the completion of the trial, indicating that the effect of both metformin and voglibose is neutral on weight homeostasis.

- Both the drugs reduced mitochondrial stress indices by decreasing glucotoxicity, though, mitophagy was significantly increased only with metformin treatment, resulting in reduced mitochondrial damage.

\section{FUNDING}

The study was supported by a grant received from the Department of Biotechnology (DBT), New Delhi, INDIA. 